\title{
MCNP6 simulation of light and medium nuclei fragmentation at intermediate energies
}

\author{
Stepan G. MashniK ${ }^{1}$ and Leslie M. Kerby ${ }^{1,2}$ \\ ${ }^{1}$ Los Alamos National Laboratory, Los Alamos, NM 87545, USA \\ ${ }^{2}$ University of Idhao, Moscow, ID 83844-4264, USA
}

\begin{abstract}
Fragmentation reactions induced on light and medium nuclei by protons and light nuclei of energies around $1 \mathrm{GeV} /$ nucleon and below are studied with the Los Alamos transport code MCNP6 and with its CEM03.03 and LAQGSM03.03 event generators. CEM and LAQGSM assume that intermediate-energy fragmentation reactions on light nuclei occur generally in two stages. The first stage is the intranuclear cascade (INC), followed by the second, Fermi breakup disintegration of light excited residual nuclei produced after the INC. CEM and LAQGSM account also for coalescence of light fragments (complex particles) up to ${ }^{4} \mathrm{He}$ from energetic nucleons emitted during INC. We investigate the validity and performance of MCNP6, CEM, and LAQGSM in simulating fragmentation reactions at intermediate energies and discuss possible ways of further improving these codes.
\end{abstract}

\section{Introduction and theoretical background}

Fragmentation reactions induced by protons and light nuclei of energies around $1 \mathrm{GeV} /$ nucleon and below on light target nuclei are involved in different applications, like cosmic-ray-induced single event upsets (SEU's), radiation protection, and cancer therapy with proton and ion beams, among others. It is impossible to measure all nuclear data needed for such applications; therefore, Monte Carlo transport codes are usually used to simulate 
impacts associated with fragmentation reactions. It is important that available transport codes simulate such reactions as well as possible.

The Los Alamos Monte Carlo transport code MCNP6 [1] uses by default the latest version of the cascade-exciton model (CEM) as incorporated in its event generator CEM03.03 to simulate fragmentation of light nuclei at intermediate energies for reactions induced by nucleons, pions, and photons, and the Los Alamos version of the quark-gluon string model (LAQGSM) as implemented in the code LAQGSM03.03 (see [2] and references therein) to simulate fragmentation reactions induced by nuclei and by particles at energies above $\sim 3.5 \mathrm{GeV}$, up to about $1 \mathrm{TeV} /$ nucleon.

Generally, both CEM and LAQGSM assume that nuclear reactions occur in three stages. The first stage is the IntraNuclear Cascade (INC), completely different in CEM and LAQGSM, in which primary particles can be re-scattered and produce secondary particles several times prior to absorption by, or escape from the nucleus. When the cascade stage of a reaction is complete, CEM and LAQGSM use the coalescence model to "create" high-energy $\mathrm{d}, \mathrm{t},{ }^{3} \mathrm{He}$, and ${ }^{4} \mathrm{He}$ via final-state interactions among emitted cascade nucleons, already outside of the target. The subsequent relaxation of the nuclear excitation is treated in terms of an improved version of the modified exciton model of preequilibrium decay followed by the equilibrium evaporation/fission stage of the reaction. But if the residual nuclei after the INC have atomic numbers with $A<13$, CEM and LAQGSM use the Fermi breakup model to calculate their further disintegration instead of using the preequilibrium and evaporation/fission models. Thus, for targets with $A<13$, reactions are assumed to occur only in two stages.

The "standard" version of CEM and LAQGSM account for possible multiple emission of only n, p, d, t ${ }^{3} \mathrm{He}$, and ${ }^{4} \mathrm{He}$ during the preequilibrium stage of reactions (see Ref. [2]). Their latest, "F", version (see Refs. [3-5]) considers a possibility of preequilibrium emission of light fragments (LF) heavier than ${ }^{4} \mathrm{He}$, up to ${ }^{28} \mathrm{Mg}$. It also simulates coalescence of LF heavier than ${ }^{4} \mathrm{He}$, up to $A=7$, in CEM03.03F (see [3-5]), and up to $A=12$, in LAQGSM03.03F (see [6]).

In recent years, MCNP6, with its CEM and LAQGSM event generators, has been extensively validated and verified $(\mathrm{V} \& \mathrm{~V})$ against a large variety of nuclear reactions on both thin and thick targets (see, e.g. Refs. [3] - [8] and references therein). In Ref. [3], it was tested specifically on fragmentation of light nuclei at intermediate energies. Here, we present a few results from our recent work [3] and investigate further the performance of MCNP6, CEM, and LAQGSM in simulating fragmentation reactions at intermediate energies and discuss possible ways of further improving these codes. 


\section{Results and conclusion}

Figs. 1, 2, 3, and 5 show examples of fragmentation reactions on light nuclei simulated by our codes. Figs. 6 and 4 shows examples for medium targets, ${ }^{48} \mathrm{Ca}$ and ${ }^{\text {nat }} \mathrm{Ag}$. Many more similar results, their discussion, and useful details can be found in Refs. [3] - [9] and references therein.
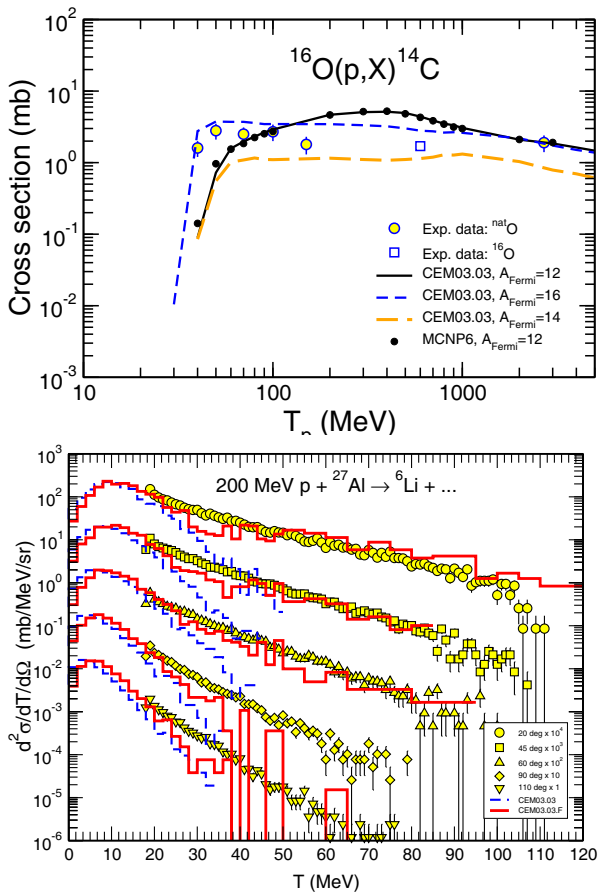

Figure 1: Excitation function for the production of ${ }^{14} \mathrm{C}$ from $\mathrm{p}+{ }^{16} \mathrm{O}$ calculated with CEM03.03 using the "standard" version of the Fermi breakup model $\left(A_{\text {Fermi }}=12\right)$ and with cutoff values for $A_{F e r m i}$ of 16 and 14 , as well as with MCNP6 using CEM03.03 $\left(A_{F e r m i}=12\right)$ compared with experimental data, as indicated. Experimental data are from the T16 Lib compilation [10] (see details in [3]).

Figure 2: Comparison of experimental ${ }^{6} \mathrm{Li}$ spectra at 20,45, 60, 90, and 110 degrees by Machner et al. [11] (symbols) with calculations by the unmodified CEM03.03 (dashed histograms) and results by CEM03.03.F (solid histograms), as indicated (see more details in $[3])$.

Our results indicate that MCNP6 using CEM03.03 and LAQGSM03.03 simulates fragmentation reactions on light and

medium-light nuclei at intermediate energies well, in a satisfactory agreement with experimental data. The recent "F" version of codes (see Refs. [3] - [6]) is even better, as it allows us to describe emission of energetic LF from practically arbitrary reactions.

However, MCNP6 is not yet ready to predict well heavy fragments from reactions with heavier nuclei, with mass numbers $A \sim 100$. Such nuclear targets are considered too light to fission in conventional codes. Similarly, the fragments are too light to be produced as spallation residues and too heavy to be produced via standard evaporation and/or preequilibrium models, or via coalescence. 

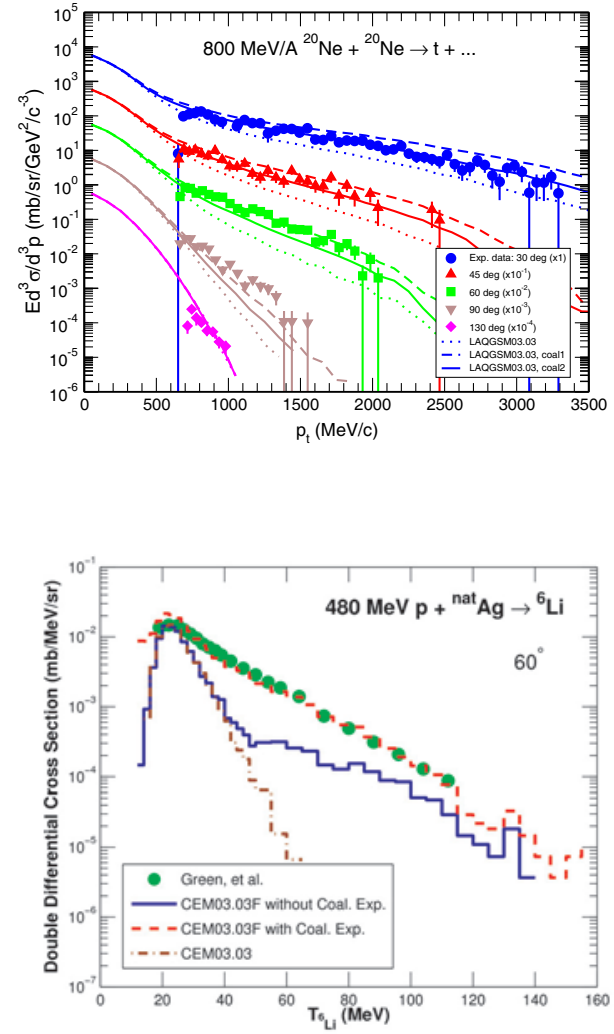

Figure 3: Comparison of measured [12] $\mathrm{t}$ spectra at 45, 60, 90, and 130 degrees from $800 \mathrm{MeV} /$ nucleon ${ }^{20} \mathrm{Ne}+$ ${ }^{20} \mathrm{Ne}$ with calculations by LAQGS03.03 using its "standard" version of the coalescence model $\left(p_{0}=0.108 \mathrm{GeV} / \mathrm{c}\right.$ for $\mathrm{t}$ and ${ }^{3} \mathrm{He}$; dotted lines) and with modified values of $p_{0}$ labeled in legend as "coal1" (dashed lines) and "coal2" (solid lines), as indicated in legend and discussed in detail in Ref. [3].

Figure 4: Comparison of experimental data by Green et al. [13] (circles) for the production of ${ }^{6} \mathrm{Li}$ at an angle of $60^{\circ}$ from the reaction 480 $\mathrm{MeV} p+{ }^{n a t} \mathrm{Ag}$, with results by standard CEM03.03 (dot-dashed line) from CEM03.03F without coalescence expansion (solid line) and CEM03.03F with coalescence expansion (dashed line) (see details in $[5,19])$.

One way to approach this problem would be to employ after the INC stage of reactions a fission-like sequential-binary-decay model, like the code GEMINI by Charity et al. [16] to describe the compound nuclear decay. In our case, this means separately merging CEM and LAQGSM with GEMINI. Actually, we already have done so more than a decade ago, producing the "G" versions of CEM and LAQGSM we had at that time (see, e.g., Ref. [18] and references therein).

Another way to address this problem is to implement in CEM and LAQGSM the Statistical Multifragmentation Model (SMM) by Botvina et al. [17]. Thus, we would consider multifragmentation as a mode competitive to evaporation of particles and light fragments, when the excitation energy $E^{*}$ of a compound nucleus produced after the preequilibrium stage of a reaction is above a certain value, $E_{t r}^{*}$, e.g., $E_{t r}^{*}=2 \times A \mathrm{MeV}$, as we did in the "S" versions of CEM03.01 and LAQGSM03.01 (see, e.g., Ref. [18] and references therein).

As of today, neither the "S" nor the "G" versions of CEM and LAQGSM have been implemented in MCNP6. We plan to incorporate them in our 
event generators used by MCNP6 after we tune several parameters in SMM and GEMINI, that are essential in chosing the excitation energy (or temper-

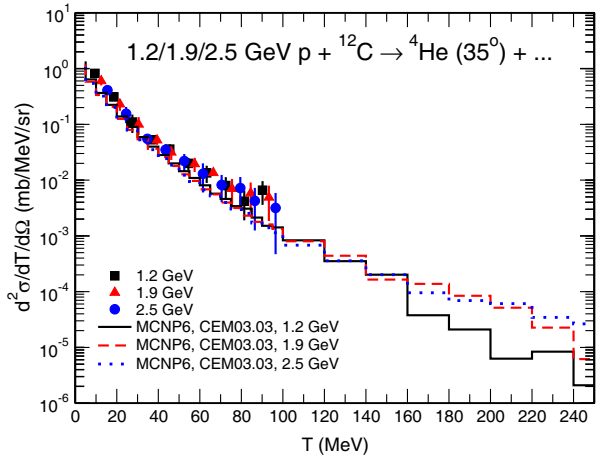

Figure 5: ${ }^{4} \mathrm{He}$ spectra $\left(\right.$ at $\left.35^{\circ}\right)$ from $1.2 / 1.9 / 2.5 \mathrm{GeV} \mathrm{p}+{ }^{12} \mathrm{C}$ measured by M. Fidelus of the PISA collaboration [14] (symbols) with calculations by MCNP6 using CEM03.03 (see details in $[5])$.

ature) of nuclei when reaction mechanisms change from "usual evaporation", to binary decays described by GEMINI, and/or to multifragmentation simulated with SMM (see details in [19]).

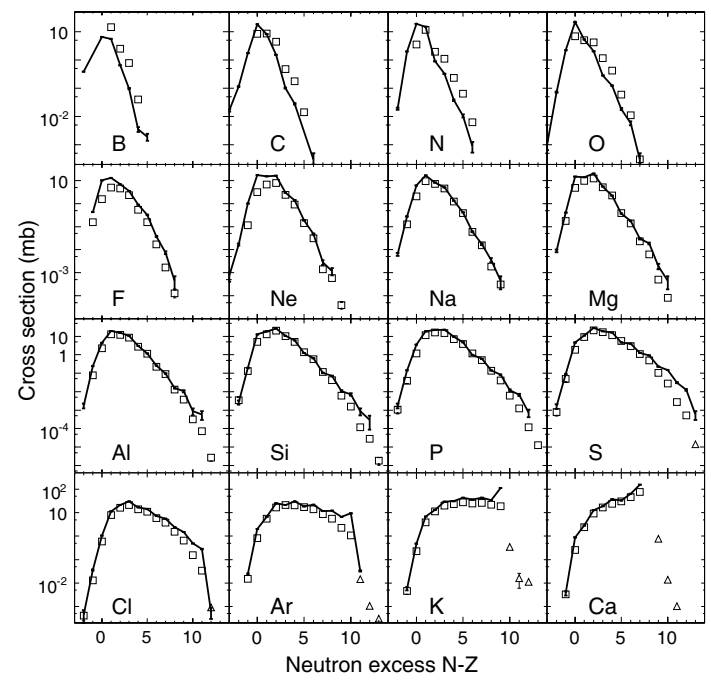

Figure 6: Measured cross sections for ${ }^{48} \mathrm{Ca}$ fragmentation on ${ }^{9} \mathrm{Be}$ at $140 \mathrm{MeV} /$ nucleon [15] compared with LAQGSM03.03 predictions (see details in [9]).

This study was carried out under the auspices of the National Nuclear Security Administration of the U.S. Department of Energy at Los Alamos National Laboratory under Contract No. DE-AC52-06NA25396. This work is supported in part (for L.M.K) by the M. Hildred Blewett Fellowship of the American Physical Society, www.aps.org. 


\section{References}

[1] T. Goorley et al. Nucl. Technol. 180 (2012) 298.

[2] S. G. Mashnik et al. CEM03.03 and LAQGSM03.03 Event Generators for the MCNP6, MCNPX, and MARS15 Transport Codes, LANL Report LA-UR-08-2931, Los Alamos (2008); arXiv:0805.0751.

[3] S. G. Mashnik and L. K. Kerby, NIM A 764 (2014) 59.

[4] L. K. Kerby and S. G. Mashnik, NIM B 356-357 (2015) 135.

[5] L. M. Kerby, Precompound emission of energetic light fragments in spallation reactions, $\mathrm{PhD}$ thesis, University of Idaho, 2015.

[6] K. K. Gudima et al., Fragmentation of Light Nuclei at Intermediate Energies Simulated with MCNP6, LANL Report LA-UR-15-20953, invited talk at NUFRA2015, 4 - 11 October 2015, Kemer, Turkey.

[7] S. G. Mashnik, Eur. Phys. J. Plus 126 (2011) 49; arXiv:1011.4978

[8] S. G. Mashnik, Validation and Verification of MCNP6 Against HighEnergy Experimental Data and Calculations by Other Codes. Primers I to IV, LANL Reports LA-UR-11-05129, LA-UR-11-05627, LAUR-13-26944, LA-UR-14-27018 Los Alamos (2011, 2013, and 2014); https://mcnp.lanl.gov/.

[9] S. G. Mashnik et al. LAQGSM03.03 Upgrade and Its Validation, LANL Report LA-UR-07-6198 Los Alamos (2007); arXiv:0709.1736.

[10] S. G. Mashnik et al. in Proc. SARE-4, Knoxville, TN, September 1316, 1998 (ORNL, 1999, pp. 151-162); arXiv:nucl-th/9812071; our T-16 Library "T-16 Lib" is updated permanently when new experimental data became available to us.

[11] H. Machner et al. Phys. Rev. C 73 (2006) 044606.

[12] M.-C. Lemaire et al. Lawrence Berkeley Laboratory Report LBL-8463 (1978).

[13] R. Green et al. Phys. Rev. C, 29 (1984) 1806.

[14] M. Fidelus, Model Description of Proton Induced Fragmentation of Atomic Nuclei, Ph.D. Thesis, Jagiellonian University, Cracow, 2010.

[15] Michal Mocko, Rare Isotope Production, PhD thesis, Michigan State University, 2006.

[16] R. J. Charity et al., Nucl. Phys. A 483 (1988) 371.

[17] J. P. Bondorf, A. S. Botvina et al., Phys. Rep. 257 (1995) 133. 
[18] S. G. Mashnik et al., CEM03.S1, CEM03.G1, LAQGSM03.S1, and LAQGSM03.G1 Versions of CEM03.01 and LAQGSM03.01 EventGenerators, LANL Report LA-UR-06-1944, Los Alamos (2006); E-print: nucl/th-0603046.

[19] S. G. Mashnik, Possible Improvements to MCNP6 and its CEM/LAQGSM Event-Generators, LANL Report LA-UR-UR-1526166, Los Alamos (2015). 